PROCEEDINGS OF THE

AMERICAN MATHEMATICAL SOCIETY

Volume 128, Number 6, Pages 1721-1728

S 0002-9939(99)05200-4

Article electronically published on October 27, 1999

\title{
KUTTNER'S PROBLEM AND A PÓLYA TYPE CRITERION FOR CHARACTERISTIC FUNCTIONS
}

\author{
TILMANN GNEITING
}

(Communicated by Christopher D. Sogge)

\begin{abstract}
Let $\varphi:[0, \infty) \rightarrow \mathbb{R}$ be a continuous function with $\varphi(0)=1$ and $\lim _{t \rightarrow \infty} \varphi(t)=0$. If $t^{-1}\left(\sqrt{t} \varphi^{\prime \prime}(\sqrt{t})-\varphi^{\prime}(\sqrt{t})\right)$ is convex, then $\psi(t)=\varphi(|t|)$, $t \in \mathbb{R}$, is the characteristic function of an absolutely continuous probability distribution. The criterion complements Pólya's theorem and applies to characteristic functions with various types of behavior at the origin. In particular, it provides upper bounds on Kuttner's function $k(\lambda), \lambda \in(0,2)$, which gives the minimal value of $\kappa$ such that $\left(1-|t|^{\lambda}\right)_{+}^{\kappa}$ is a characteristic function. Specifically, $k(5 / 3) \leq 3$. Furthermore, improved lower bounds on Kuttner's function are obtained from an inequality due to Boas and Kac.
\end{abstract}

\section{INTRODUCTION}

One of the most beautiful results in classical analysis and probability theory is the celebrated criterion of Pólya [8].

Theorem 1.1 (Pólya). Let $\varphi:[0, \infty) \rightarrow \mathbb{R}$ be a continuous function with $\varphi(0)=1$ and $\lim _{t \rightarrow \infty} \varphi(t)=0$. If $\varphi$ is convex, then $\psi(t)=\varphi(|t|), t \in \mathbb{R}$, is the characteristic function of an absolutely continuous, symmetric probability distribution 1

Sasvári 9 provides interesting comments and a simple proof of this result. Pólya's criterion also has a wealth of applications, for which we refer to Feller (3), Chapter XV) and Lukacs ([6], Section 4.3). However, characteristic functions of Pólya type are not differentiable at $t=0$. In view of the well-known relationship between the differentiability of characteristic functions and the existence of moments for probability distributions, they correspond to probability measures with infinite variance. Our key result here is an analogue of Pólya's criterion that also applies to characteristic functions $\psi(t)=\varphi(|t|)$, which are twice differentiable at $t=0$ and correspond to probability distributions with finite variance $-\psi^{\prime \prime}(0)$. Before we present the new criterion, let us recall that a characteristic function $\psi$ for which $\psi^{\prime \prime}(0)$ exists is twice differentiable everywhere (Lukacs [6], Theorem 2.3.1). Thus, we expect the analogue to put a condition on the second derivative of $\varphi$.

Received by the editors July 13, 1998.

1991 Mathematics Subject Classification. Primary 42A82, 60E10; Secondary 42A24, 42A38.

${ }^{1}$ As in Sasvári [9], we use the terminology of probability theory, where Pólya's criterion seems to have most of its applications. In the language of analysis, $\psi$ is the Fourier transform of an even and nonnegative function, which integrates to 1 over the real line. In particular, $\psi$ is positive definite. 
Theorem 1.2. Let $\varphi:[0, \infty) \rightarrow \mathbb{R}$ be a continuous function with $\varphi(0)=1$ and $\lim _{t \rightarrow \infty} \varphi(t)=0$. If $\varphi$ has two continuous derivatives and

$$
\frac{1}{t}\left(\sqrt{t} \varphi^{\prime \prime}(\sqrt{t})-\varphi^{\prime}(\sqrt{t})\right)
$$

is convex, then $\psi(t)=\varphi(|t|), t \in \mathbb{R}$, is the characteristic function of an absolutely continuous, symmetric probability distribution.

Whenever the function $\varphi:[0, \infty) \rightarrow \mathbb{R}$ has four continuous derivatives on $(0, \infty)$, the convexity of (1) is equivalent to the nonnegativity of

$$
t^{3} \varphi^{\prime \prime \prime \prime}(t)-4 t^{2} \varphi^{\prime \prime \prime}(t)+8 t \varphi^{\prime \prime}(t)-8 \varphi^{\prime}(t)
$$

for $t>0$. This condition can be checked very efficiently.

Example 1.3. Straightforward evaluation of the derivatives in (2) shows that each of the following is a characteristic function: (i) $\psi(t)=\ln ((|t|+e) /(|t|+1)$ ); (ii) $\psi(t)=1 /(1+|t|)^{\alpha}, \alpha \geq 0$; (iii) $\psi(t)=1 /\left(1+|t|^{\beta}\right), 0<\beta \leq 1.877$; (iv) $\psi(t)=$ $\left(1+|t|+\gamma t^{2}\right) \exp (-|t|), 0 \leq \gamma \leq 1 / 4 ;(\mathrm{v}) \psi(t)=(1-|t|)_{+}^{3}(1+3|t|)$.

The functions in (i) and (ii) are convex on $(0, \infty)$, such that Pólya's criterion applies, too. For Linnik's function (iii), $\psi(t)=1-|t|^{\beta}+\mathcal{O}\left(|t|^{2 \beta}\right)$ as $t \rightarrow 0$, and Theorem 1.1 applies only if $\beta \leq 1$. In cases (iv) and (v), $\psi$ has two continuous derivatives and corresponds to a probability density function with finite variance. Pólya's criterion does not apply to this type of candidate function, but Theorem 1.2 works.

Section 2 develops the motivation for and the proof of Theorem 1.2. The general idea is that an analogue of Pólya's criterion will provide necessary and sufficient conditions for a scale mixture representation in terms of a suitable basis function.

In Section 3, we consider Wintner's [12] and Kuttner's [5] question for which values of $\lambda>0$ and $\kappa>0$ we can identify

$$
\psi_{\lambda, \kappa}(t)=\left(1-|t|^{\lambda}\right)_{+}^{\kappa}
$$

as a characteristic function. Here, $u_{+}^{\kappa}$ stands for $u^{\kappa}$ or 0 according as to $u>0$ or $u \leq 0$, respectively. It was known to Wintner and Kuttner that $\lambda<2$ is a necessary condition, regardless of the value of $\kappa>0$. Furthermore, Kuttner showed that there exists a function $k(\lambda), \lambda \in(0,2)$, such that $\psi_{\lambda, \kappa}$ is a characteristic function if and only if $\kappa \geq k(\lambda)$. The function $k(\lambda)$ is continuous and strictly increasing, $\lim _{\lambda \rightarrow 0} k(\lambda)>0, k(1)=1, \lim _{\lambda \rightarrow 2} k(\lambda)=\infty$, and $k(\lambda)>\lambda$ if $\lambda \neq 1$. Theorems 3.1 and 3.2 and Tables 1 and 2 respectively, sharpen Kuttner's results. Lower estimates for $k(\lambda)$ follow from an inequality of Boas and Kac [2], and Theorem 1.2 provides upper bounds for Kuttner's function and $\lambda \leq 1.8252$.

\section{IDEA AND PROOF OF THE CRITERION}

The idea of Theorem 1.2 stems from an attractive interpretation of Pólya's criterion (compare Sasvári [9]): Theorem 1.1 gives necessary and sufficient conditions for a function $\psi$ to be of the form

$$
\psi(t)=\int_{(0, \infty)}(1-|r t|)_{+} d F(r), \quad t \in \mathbb{R}
$$

where $F$ is a probability measure on $(0, \infty)$. In other words, $\psi$ has a representation as a scale mixture of $\psi_{0}(t)=(1-|t|)_{+}$with respect to $F$. Expressed in terms of random variables, $\psi$ is the characteristic function of $R X$, where $X$ has characteristic 
function $\psi_{0}(t)$ or density $p_{0}(x)=(1-\cos x) /\left(\pi x^{2}\right), R$ has distribution $F$, and $X$ is independent of $R$. This observation is the key to Theorem 1.2. We will take up the scale mixture approach and apply it to the characteristic function

$$
\psi_{1}(t)=(1-|t|)_{+}^{3}\left(1+3|t|+t^{2}\right) .
$$

This function has recently been discussed by $\mathrm{Wu}$ [13 in the context of radial basis function interpolation. It is the self-convolution of $\sqrt{30} / 4\left(1-4 u^{2}\right)_{+}$, and the corresponding probability density function is

$$
p_{1}(x)=\frac{60}{\pi x^{6}}\left(x \cos \left(\frac{x}{2}\right)-2 \sin \left(\frac{x}{2}\right)\right)^{2} .
$$

The proof of Theorem 1.2 will now be immediate from the following result.

Theorem 2.1. Theorem 1.2 gives necessary and sufficient conditions for a function $\psi$ to be of the form

$$
\psi(t)=\int_{(0, \infty)} \psi_{1}(r t) d F(r), \quad t \in \mathbb{R},
$$

where $\psi_{1}$ is the characteristic function (3) and $F$ is a probability measure on $(0, \infty)$.

Proof. Suppose $\psi$ is of the form (15) and denote by $\varphi$ its restriction to $[0, \infty)$. Then clearly $\varphi(0)=1, \lim _{t \rightarrow \infty} \varphi(t)=0$, and $\varphi$ has two continuous derivatives on $(0, \infty)$. Furthermore,

$$
\frac{1}{t}\left(\sqrt{t} \varphi^{\prime \prime}(\sqrt{t})-\varphi^{\prime}(\sqrt{t})\right)=15 \int_{(0, \infty)}\left(1-r^{2} t\right)+r^{3} d F(r)
$$

is convex by Theorem 1 of Williamson [11.

Conversely, suppose $\varphi:[0, \infty) \rightarrow \mathbb{R}$ satisfies the conditions of Theorem 1.2 Then

$$
\theta(t)=\frac{1}{t}\left(\sqrt{t} \varphi^{\prime \prime}(\sqrt{t})-\varphi^{\prime}(\sqrt{t})\right)=\left.\frac{d}{d u}\left(\frac{\varphi^{\prime}(u)}{u}\right)\right|_{u=\sqrt{t}}
$$

is convex on $(0, \infty)$. Thus, $\theta(t)$ tends to some, possibly infinite, limit as $t \rightarrow \infty$, and so does $\theta\left(t^{2}\right)=d / d t\left[\varphi^{\prime}(t) / t\right]$. Since $\lim _{t \rightarrow \infty} \varphi(t)=0$, the only possible limit is 0 . Therefore, $\theta(t)$ is convex and decreases to 0 on $(0, \infty)$. By Theorems 1 and 3 of Williamson [11, $\theta(t)$ is of the form

$$
\theta(t)=\int_{(0, \infty)}(1-r t)_{+} d G(r), \quad t>0,
$$

where $G(r)=\theta(1 / r)-1 / r \theta^{\prime}(1 / r)$ is nondecreasing. Combining Equations (6) and (17), and replacing $t$ by $t^{2}$, leads to

$$
\begin{aligned}
\left.\frac{d}{d u}\left(\frac{\varphi^{\prime}(u)}{u}\right)\right|_{u=t} & =\int_{(0, \infty)}\left(1-r^{2} t^{2}\right)+d G\left(r^{2}\right) \\
& =\left.\int_{(0, \infty)} \frac{d}{d u}\left(\frac{r \varphi_{1}^{\prime}(r u)}{u}\right)\right|_{u=t} d F(r), \quad t>0
\end{aligned}
$$

where $d F(r)=d G\left(r^{2}\right) /\left(15 r^{3}\right)$, and where $\varphi_{1}$ denotes the restriction of $\psi_{1}$ to $[0, \infty)$. Since $\varphi(0)=1$ and $\lim _{t \rightarrow \infty} \varphi(t)=0$, two-fold integration with respect to $t$ and an application of Fubini's theorem show that

$$
\varphi(t)=\int_{(0, \infty)} \varphi_{1}(r t) d F(r), \quad t \geq 0 .
$$


Furthermore, $F$ is a probability measure, because $\varphi(0)=1$. Writing (8) in terms of $\psi$ and $\psi_{1}$, we see that (5) holds.

Proof of Theorem 1.2] Suppose $\varphi:[0, \infty) \rightarrow \mathbb{R}$ satisfies the conditions of the theorem. Theorem 2.1 shows that $\psi(t)=\varphi(|t|)$ is of the form (50) for some probability measure $F$. Thus, $\psi$ is the characteristic function of the random variable $R X$, where $X$ has characteristic function (3) or density (4), $R$ has distribution $F$, and $X$ is independent of $R$. Since $X$ is absolutely continuous and symmetric, $R X$ is such, too.

Corollary 2.2. Suppose $\psi(t)=\varphi(|t|)$ is of the form (5). Then $F$ is given by

$$
d F(r)=\left.\frac{1}{15 r^{3}} d\left(\theta(t)-t \theta^{\prime}(t)\right)\right|_{t=1 / r^{2}}, \quad r>0,
$$

where $\theta(t)$ is defined by (6). Moreover, $\psi$ is the Fourier transform of the probability density function

$$
p(x)=\frac{60}{\pi x^{6}} \int_{(0, \infty)}\left(\frac{x}{r} \cos \left(\frac{x}{2 r}\right)-2 \sin \left(\frac{x}{2 r}\right)\right)^{2} r^{5} d F(r) .
$$

Proof. Equation (9) follows from the proof of Theorem 2.1; (10) is a consequence of the stochastic representation in the proof of Theorem 1.2 and the formula for the density of products of independent random variables.

The preceding discussion clearly calls for further analogues of Pólya's criterion. The idea is to take a characteristic function of the form

$$
\psi(t)= \begin{cases}q(|t|), & |t| \leq 1 \\ 0, & \text { otherwise }\end{cases}
$$

where $q$ is a low-degree polynomial, and to characterize its scale mixtures in analogy to Theorem 2.1. Characteristic functions of this type have recently been studied by Wendland 10 and $\mathrm{Wu} 13$. Wendland showed that if $\psi$ is a characteristic function of the form (11) with $2 k$ continuous derivatives, then the degree of the polynomial $q$ must be at least $3 k+1$. Moreover, there exists exactly one such function with minimal degree $3 k+1$. If $k=0$, this unique function is $\psi_{0}(t)=(1-|t|)_{+}$, and characterizing its scale mixtures leads to Pólya's criterion. If $k=2$, Wendland's function of minimal degree is that of Example 1.3(v). This explains why Theorem 1.2 works with Wu's function (3), which is of higher degree. Since Wendland's function is a scale mixture of Wu's function, an application of the scale mixture approach to the former must lead to a weaker criterion. Specifically, the criterion based on Wendland's function requires the function in (1) with $t$ replaced by $t^{2}$ to be convex. This criterion is evidently weaker, but not of a simpler form. The same conclusion holds for all the functions in the tables of Wendland [10] and $\mathrm{Wu}[13$. which have two, but not four, continuous derivatives.

Thus, we expect stronger or complementary criteria of the Pólya type to base on characteristic functions of the form (11) which have four or more continuous derivatives. Then the corresponding polynomials $q$ will be of degree at least 7 , and sufficient conditions in analogy to (11) and (2) will involve derivatives of orders at least 4 and 6 , respectively. In other words, Theorem 1.2 may provide the optimal balance between a wide range of applicability and a simple formulation in the spirit of Pólya's original criterion. 


\section{KutTNER'S PROBLEM}

In 1942, Wintner [12] asked the interesting question for which values of $\lambda>0$ and $\kappa>0$ we can identify

$$
\psi_{\lambda, \kappa}(t)=\left(1-|t|^{\lambda}\right)_{+}^{\kappa}
$$

as a characteristic function. His question is an appealing special case of a recent problem of Bisgaard and Sasvári [1] on positive definite functions. By Fourier inversion, $\psi_{\lambda, \kappa}$ is a characteristic function if and only if

$$
\int_{0}^{1}\left(1-t^{\lambda}\right)^{\kappa} \cos (x t) d t \geq 0, \quad x>0 .
$$

Rephrased this way, the problem superficially looks simple, but it is not. A first observation is that $\psi_{\lambda, \kappa}(t)=1-\mathcal{O}\left(|t|^{\lambda}\right)$ as $t \rightarrow 0$. For a characteristic function, this is possible only if $\lambda \leq 2$. If $\lambda=2$, the classical integral representation of the Bessel function $J_{\kappa+1 / 2}(x) / x^{\kappa+1 / 2}$ shows that (12) changes sign as a function of $x$. The difficulties come with the case $\lambda<2$. Wintner claimed that if $\kappa \geq 1$, then $\psi_{\lambda, \kappa}$ is a characteristic function if and only if $\lambda \leq 1$. Two years later, Kuttner [5] approached the same problem in the context and language of summability theory. Apparently unaware of Wintner's work, Kuttner claimed that there exists a function $k(\lambda), \lambda \in(0,2)$, such that $\psi_{\lambda, \kappa}$ is a characteristic function if and only if $\kappa \geq$ $k(\lambda)$. The function $k(\lambda)$ is continuous and strictly increasing, $\lim _{\lambda \rightarrow 0} k(\lambda)>0$, $k(1)=1, \lim _{\lambda \rightarrow 2} k(\lambda)=\infty$, and $k(\lambda)>\lambda$ if $\lambda \neq 1$. Kuttner's findings clearly contradict Wintner's earlier claim. Yet they are correct, and they certainly justify the association of the problem with Kuttner's name. Note that Wintner ([12], footnote 5 on p. 307) supports his assertion merely by the statement that it "is easy to transfer this primitive proof [...] if use is made of the second mean-value theorem." Kuttner's results and Theorem 3.2 below tell a different story.

Since Kuttner's work, little progress has been made, and upper bounds for $k(\lambda)$ and $\lambda>1$ have not been found. The only related result of which the author knows is Proposition 2.6 of Misiewicz and Richards [7]. It states that there exists a function $k_{0}(\lambda), \lambda \in(0,1)$, such that

$$
\int_{0}^{1}\left(1-t^{\lambda}\right)^{\kappa} \cos (3 \pi t / 2) d t \quad\left\{\begin{array}{cc}
<0, & \kappa<k_{0}(\lambda) \\
>0, & \kappa>k_{0}(\lambda) .
\end{array}\right.
$$

Then evidently $k(\lambda) \geq k_{0}(\lambda)$, and the numerical values in Table 1 of Misiewicz and Richards [7] sharpen Kuttner's lower estimate if $0.1 \leq \lambda<0.519$. Zastavnyi's recent work [14] contains some beautiful results on positive definite functions and calls for explicit upper bounds for Kuttner's function. The results below have been motivated by his work. Theorem 3.1 gives improved lower bounds, and Theorem 3.2 provides an upper estimate for $\lambda \leq 5 / 3$. Numerical values are given in Tables

\begin{tabular}{|c|c|c|c|c|c|c|c|c|c|c|}
\hline $\bar{\lambda}$ & $\overline{0.05}$ & $\overline{0.15}$ & $\overline{0.25}$ & $\overline{0.35}$ & $\overline{0.45}$ & 0.55 & 0.65 & 0.75 & 0.85 & 0.95 \\
\hline$l(\lambda)$ & 0.2051 & 0.2993 & 0.3770 & 0.4515 & 0.5263 & 0.6033 & 0.6834 & 0.7676 & 0.8565 & 0.9507 \\
\hline$\lambda$ & 1.05 & 1.15 & 1.25 & 1.35 & 1.45 & 1.55 & 1.65 & 1.75 & 1.85 & 1.95 \\
\hline$l(\lambda)$ & 1.0507 & 1.1572 & 1.2706 & 1.3916 & 1.5247 & 1.7234 & 1.9795 & 2.3462 & 2.9103 & 3.9084 \\
\hline
\end{tabular}
[1] and 2 .

TABLE 1. Lower bound $l(\lambda)$ for Kuttner's function. 
TABLE 2. Lower and upper bounds $l(\lambda)$ and $u(\lambda)$, respectively, for Kuttner's function.

\begin{tabular}{|c|c|c|c|c|c|c|}
\hline$\lambda$ & $5 / 3$ & 1.7908 & 1.8085 & 1.8169 & 1.8219 & 1.8252 \\
\hline$l(\lambda)$ & 2.0301 & 2.5454 & 2.6434 & 2.6932 & 2.7232 & 2.7441 \\
\hline$u(\lambda)$ & 3.0000 & 4.0000 & 5.0000 & 6.0000 & 7.0000 & 8.0000 \\
\hline
\end{tabular}

Theorem 3.1. Let $k(\lambda), \lambda \in(0,2)$, denote Kuttner's function. Then

$$
k(\lambda) \geq l_{n}(\lambda)=-\frac{\ln (\cos (\pi /(n+1)))}{\lambda \ln (n)-\ln \left(n^{\lambda}-1\right)}, \quad \lambda \in(0,2), \quad n=2,3, \ldots .
$$

Proof. Let $n \geq 2$ be a positive integer. By Theorem 2 of Boas and Kad2 [2], a necessary condition on $\psi_{\lambda, \kappa}$ to be a characteristic function is that

$$
\psi_{\lambda, \kappa}\left(\frac{1}{n}\right)=\left(\frac{n^{\lambda}-1}{n^{\lambda}}\right)^{\kappa} \leq \cos \left(\frac{\pi}{n+1}\right) .
$$

Solving the inequality for $\kappa$ leads to (13).

Table 1 displays lower bounds for Kuttner's function that result from (13). For $\lambda \leq 1.35$, the maximal bound in (13) occurs at $n=2$; for $\lambda=1.45$ and 1.55 at $n=3$; and for $\lambda=1.65,1.75,1.85$, and 1.95 , at $n=4,6,12$, and 38 , respectively. The numerical values generally sharpen Kuttner's estimate $k(\lambda)>\lambda$. However, the approach does not lead to a positive lower bound for $\lim _{\lambda \rightarrow 0} k(\lambda)$, because, for each $n, \lim _{\lambda \rightarrow 0} l_{n}(\lambda)=0$. Furthermore, $\lim _{n \rightarrow \infty} \lim _{\lambda \rightarrow 2} l_{n}(\lambda)=\pi^{2} / 2$ is finite.

Theorem 3.2. Let $\varphi_{\lambda, 3}(t)=\left(1-t^{\lambda}\right)_{+}^{3}, t \in[0, \infty)$. Then $\varphi_{\lambda, 3}$ satisfies the conditions of Theorem 1.2 if and only if $\lambda \leq 5 / 3$.

Proof. We need to check whether

$$
\begin{aligned}
\theta_{\lambda, 3}(t) & =\frac{1}{t}\left(\sqrt{t} \varphi_{\lambda, 3}^{\prime \prime}(\sqrt{t})-\varphi_{\lambda, 3}^{\prime}(\sqrt{t})\right) \\
& =3 \lambda t^{(\lambda-3) / 2}\left((2-\lambda)-(2-3 \lambda) t^{\lambda / 2}\right)\left(1-t^{\lambda / 2}\right)_{+}
\end{aligned}
$$

is convex. This function has a continuous second derivative

$$
\theta_{\lambda, 3}^{\prime \prime}(t)=\frac{3}{4} \lambda t^{(\lambda-7) / 2} q_{\lambda}\left(t^{\lambda / 2}\right)
$$

on $(0,1)$, where $q_{\lambda}$ is a polynomial of degree 2 whose coefficients are functions of $\lambda$. Elementary calculations show that $q_{\lambda}$ is nonnegative on $(0,1)$ if and only if $\lambda \leq 5 / 3$.

In particular, $k(5 / 3) \leq 3$ for Kuttner's function, and $\psi_{\lambda, \kappa}$ is a characteristic function if $\lambda \leq 5 / 3$ and $\kappa \geq 3$. The same approach applied to the general function

$$
\varphi_{\lambda, \kappa}(t)=\left(1-t^{\lambda}\right)_{+}^{\kappa}
$$

leads to further upper bounds for $k(\lambda)$. To apply Theorem 1.2 we must require that $\kappa>2$, such that $\varphi_{\lambda, \kappa}$ is twice differentiable. Then the condition to be checked is whether

$$
\theta_{\lambda, \kappa}(t)=\kappa \lambda t^{(\lambda-3) / 2}\left((2-\lambda)-(2-\kappa \lambda) t^{\lambda / 2}\right)\left(1-t^{\lambda / 2}\right)_{+}^{\kappa-2}
$$

\footnotetext{
${ }^{2}$ There is an error in the original statement of Theorem 2 by Boas and Kac [2], which is corrected in the errata (Duke Math. J. 15 (1948), 107).
} 
is convex. Taking the second derivative of this function, the problem reduces to the question whether a polynomial of degree at most 3 remains nonnegative on $(0,1)$. Rather than giving the tedious details, we summarize some of the resulting upper bounds on Kuttner's function in Table 2. The table also shows the corresponding lower bound obtained from (13), and two observations are striking. Theorem 1.2 does not seem to apply to $\varphi_{\lambda, \kappa}$ if $\kappa<3$, and the gap between the lower and upper bound widens as $\lambda$ increases. The following proposition provides some explanation.

Proposition 3.3. If $\kappa<3$ or $\lambda \geq 1.84171$, then $\varphi_{\lambda, \kappa}(t)=\left(1-t^{\lambda}\right)_{+}^{\kappa}$ does not satisfy the conditions of Theorem 1.2 .

Proof. If $\kappa \leq 2$, then $\varphi_{\lambda, \kappa}(t)$ is not twice differentiable at $t=1$. If $\kappa \in(2,3)$, it is easily seen from (14) that $\lim _{t \rightarrow 1-} \theta_{\kappa, \lambda}^{\prime}(t)=-\infty$. Thus, $\theta_{\lambda, \kappa}$ is not convex, and the assumptions of Theorem 1.2 are violated.

The assertion for $\lambda \geq \lambda_{0}=1.84171$ is proved by contradiction. Suppose $\varphi_{\lambda, \kappa}$ satisfies the conditions of Theorem 1.2 for some $\lambda \geq \lambda_{0}$ and $\kappa>0$. In view of Theorem 2.1] every scale mixture of $\varphi_{\lambda, \kappa}$ will satisfy the conditions of Theorem 1.2 too. Now Proposition 2.2 of Keilson and Steutel [4] and Williamson's [11] results imply that $\exp \left(-t^{\lambda_{0}}\right)$ admits a representation as a scale mixture of $\varphi_{\lambda, \kappa}$. This is the desired contradiction, because if $\varphi(t)=\exp \left(-t^{\lambda_{0}}\right)$, then (2) attains negative values in a neighborhood of $t=0.674$.

Let us note here that $\varphi(t)=\exp \left(-t^{\beta}\right)$ satisfies the conditions of Theorem [1.2 if $\beta \leq 1.84170$. The proposition shows that Table 2 basically covers the range of $\lambda$ for which Theorem 1.2 provides upper bounds for Kuttner's function. Are tighter bounds within reach? A promising approach to improved lower bounds is by extensive numerical evaluation of the integrals (12) in the spirit of Misiewicz and Richards (7], Table 1). If use is made of computer algebra systems, higher-order criteria of the Pólya type, as discussed in Section 2. may eventually result in upper bounds for $\lambda>1.84170$. In any case, precise values for Kuttner's function $k(\lambda)$, $\lambda \neq 1$, remain a challenging open problem.

\section{ACKNOWLEDGEMENTS}

Much of this work has been inspired by Peter J. Huber's questions for an analogue of Pólya's criterion that applies to possibly non-convex functions. Donald Richards kindly provided many helpful and encouraging comments during the author's visit at the University of Virginia; Martin Schlather's remarks have greatly improved an earlier version of the manuscript; and Victor Zastavnyi brought Kuttner's work [5] to my attention. I am grateful to them all.

\section{REFERENCES}

[1] T. M. Bisgaard and Z. Sasvári, On the positive definiteness of certain functions, Math. Nachr. 186 (1997), 81-99. MR 99c:42009

[2] R. P. Boas and M. Kac, Inequalities for Fourier transforms of positive functions, Duke Math. J. 12 (1945), 189-206. MR 6:265h

[3] W. Feller, An introduction to probability theory and its applications, vol. II, second ed., John Wiley, New York, 1971. MR 42:5292

[4] J. Keilson and W. Steutel, Mixtures of distributions, moment inequalities and measures of exponentiality and normality, Ann. Probability 2 (1974), 112-130. MR 50:8651

[5] B. Kuttner, On the Riesz means of a Fourier series (II), J. London Math. Soc. 19 (1944), 77-84. MR 7:59d

[6] E. Lukacs, Characteristic functions, second ed., Griffin, London, 1970. MR 49:11595 
[7] J. K. Misiewicz and D. St. P. Richards, Positivity of integrals of Bessel functions, SIAM J. Math. Anal. 25 (1994), 596-601. MR 95i:33004]

[8] G. Pólya, Remarks on characteristic functions, Proceedings of the Berkeley Symposium on Mathematical Statistics and Probability (J. Neyman, ed.), University of California Press, 1949, pp. 115-123. MR 10:463c

[9] Z. Sasvári, On a classical theorem in the theory of Fourier integrals, Proc. Amer. Math. Soc. 126 (1998), 711-713. MR 98i:60013

[10] H. Wendland, Piecewise polynomial, positive definite and compactly supported radial functions of minimal degree, Adv. Comput. Math. 4 (1995), 389-396. MR 96h:41025

[11] R. E. Williamson, Multiply monotone functions and their Laplace transforms, Duke Math. J. 23 (1956), 189-207. MR 17:1061d

[12] A. Wintner, On a family of Fourier transforms, Bull. Amer. Math. Soc. 48 (1942), 304-308. MR 3:232a

[13] Z. Wu, Compactly supported positive definite radial functions, Adv. Comput. Math. 4 (1995), 283-292. MR 97g:65031

[14] V. P. Zastavnyi, On positive definiteness of some functions, Manuscript, Donetsk State University, Donetsk, Ukraine, 1998.

Department of Statistics, Box 354322, University of Washington, Seattle, WashingTON 98195

E-mail address: tilmann@stat.washington.edu 